\title{
Levosimendan Enhances Force Generation of Diaphragm Muscle from Patients with Chronic Obstructive Pulmonary Disease
}

\author{
Hieronymus W. H. van Hees ${ }^{1,3}$, P. N. Richard Dekhuijzen ${ }^{1,3}$, and Leo M. A. Heunks $1,2,3$ \\ ${ }^{1}$ Department of Pulmonary Diseases, ${ }^{2}$ Intensive Care Medicine, and ${ }^{3}$ Institute for Fundamental and Clinical Human Movement Sciences, \\ Radboud University Nijmegen Medical Centre, Nijmegen, The Netherlands
}

\begin{abstract}
Rationale: Levosimendan is clinically used to improve myocardial contractility by enhancing calcium sensitivity of force generation. The effects of levosimendan on skeletal muscle contractility are unknown. Patients with chronic obstructive pulmonary disease (COPD) suffer from diaphragm weakness, which is associated with decreased calcium sensitivity.

Objectives: To investigate the effects of levosimendan on contractility of diaphragm fibers from patients with COPD.

Methods: Muscle fibers were isolated from diaphragm biopsies obtained from thoracotomized patients with and without COPD (both groups $n=5,10$ fibers per patient). Diaphragm fibers were skinned and activated with solutions containing incremental calcium concentrations and $10 \mu \mathrm{M}$ levosimendan or vehicle $(0.02 \%$ dimethyl sulfoxide). Developed force was measured at each step and force versus calcium concentration relationships were derived. Results were grouped per myosin heavy chain isoform, which was determined by sodium dodecyl sulfate polyacrylamide gel electrophoresis (SDS-PAGE).

Measurements and Main Results: At sub-maximal activation levosimendan improved force generation of COPD and non-COPD diaphragm fibers by approximately $25 \%$, both in slow and fast fibers. Levosimendan increased calcium sensitivity of force generation $(P<$ 0.01 ) in both slow and fast diaphragm fibers from patients with and without COPD, without affecting maximal force generation.

Conclusions: Levosimendan enhances force generating capacity of diaphragm fibers from patients with and without COPD patients by increasing calcium sensitivity of force generation. These results provide a strong rationale for testing the effect of calcium sensitizers on respiratory muscle dysfunction in patients with COPD.
\end{abstract}

Keywords: diaphragm; chronic obstructive pulmonary disease; calcium sensitization; levosimendan

Weakness of the diaphragm frequently occurs in patients with chronic obstructive pulmonary disease (COPD) $(1,2)$. Inspiratory muscle weakness in these patients is associated with prolonged mechanical ventilation and increased mortality $(3,4)$. The pathophysiology of inspiratory muscle dysfunction in COPD is complex (1). Studies have shown that, in patients with COPD, impaired contractility of muscle fibers from the diaphragm, the most prominent inspiratory muscle, results from a loss of contractile protein and impaired function of remaining contractile proteins (5-7). Diaphragm muscle fibers from these patients exhibit reduced ability to respond to calcium, indicating reduced

(Received in original form May 14, 2008; accepted in final form October 23, 2008) Supported by the Department of Pulmonary Diseases, Radboud University Nijmegen Medical Centre, Nijmegen, The Netherlands

Correspondence and requests for reprints should be addressed to Leo M. A. Heunks, M.D. Ph.D., Dept of Intensive Care Medicine (551), University Medical Centre Nijmegen, PO Box 9101, 6500 HB Nijmegen, The Netherlands. E-mail: L.Heunks@ic.umcn.nl

Am J Respir Crit Care Med Vol 179. pp 41-47, 2009

Originally Published in Press as DOI: 10.1164/rccm.200805-7320C on October 31, 2008

Internet address: www.atsjournals.org

\section{AT A GLANCE COMMENTARY}

Scientific Knowledge on the Subject

Loss of contractile protein and impaired calcium sensitivity of force generation contribute to respiratory muscle dysfunction in patients with chronic obstructive pulmonary disease (COPD). No specific treatment exists to improve respiratory muscle function in these patients.

What This Study Adds to the Field

The calcium sensitizer levosimendan improves contractile protein function in diaphragm muscle fibers of patients with COPD.

calcium sensitivity of force generation. Based on these findings, we proposed that pharmacological modulation of the regulatory proteins is an effective strategy to improve diaphragm contractility in patients with COPD.

Calcium sensitizers enhance the binding of calcium to regulatory proteins of the contractile apparatus. This improves the responsiveness of myofilaments to calcium, resulting in a greater amount of force for the same amount of cytosolic calcium. An advantage of this mechanism of action is that force generation is improved without increased adenosine triphosphate (ATP) consumption (8). Currently, levosimendan is the only calcium sensitizer approved for use in human $(\sim 40$ countries worldwide). Levosimendan has been shown to improve cardiac contractility in vivo (9) and in vitro (10), by enhancing calcium sensitivity of force generation through binding with cardiac troponin C (11). The effects of calcium sensitizers on human peripheral or respiratory skeletal muscle have not been investigated. Nevertheless, cardiac troponin $\mathrm{C}$ isoform is expressed in human diaphragm muscle. Moreover, Levine and colleagues have recorded increased expression of this isoform in the diaphragm of patients with COPD (12). Therefore, we hypothesize that levosimendan improves calcium sensitivity of muscle fibers isolated from the diaphragm of patients with COPD.

\section{METHODS}

\section{Subjects}

Diaphragm muscle biopsies ( $100 \mathrm{mg}$ ) were obtained from five patients with COPD (four males) and five subjects without COPD (two males). Biopsies from the right anterior costal diaphragm were obtained during thoracotomy for lung cancer (stage $T_{1-3} \mathrm{~N}_{0-1} \mathrm{M}_{\mathrm{x}}$ ). Stages of lung cancer did not significantly differ between patients with COPD and those without COPD. Patients with chronic corticosteroid use, primary myopathies, endocrine diseases, and more than $10 \%$ weight loss in the last 6 months before surgery were excluded from the study. General characteristics and pulmonary function data are shown in Table 1. Informed consent was obtained from each subject. The 
study was approved by the Ethics Committee of Radboud University Nijmegen Medical Centre.

\section{Single Fiber Contractile Measurements}

According to previously described methods $(5,13)$, single fiber contractile measurements and experimental protocol were performed as follows.

\section{Single Fiber Preparation}

A bundle was dissected from the central part of the fresh muscle biopsy. The muscle bundle was pinned to cork and stored at $4^{\circ} \mathrm{C}$ in a relaxing solution, containing $50 \%$ glycerol (vol/vol). After 24 hours, the muscle strip was stored at $-20^{\circ} \mathrm{C}$ for later analysis. Approximately 1 hour before determination of single fiber contractile properties, the muscle bundle was transferred to relaxing solution $\left(5^{\circ} \mathrm{C}\right)$ containing $1 \%$ Triton X-100 to permeabilize lipid membranes. From the muscle bundle, approximately 2-mm segments of single fibers were isolated using micro-forceps. Subsequently, the fiber ends were attached to aluminum foil clips and mounted on the single fiber apparatus. Fibers were mounted in a temperature-controlled $\left(20^{\circ} \mathrm{C}\right)$ flow-through acrylic chamber (120- $\mu$ l volume), with a glass coverslip bottom, on the stage of an inverted microscope (model IX-70; Olympus, Amsterdam, The Netherlands).Two stainless steel hooks were used to mount the fiber horizontally in the chamber. One end of the fiber was attached to a force transducer (model AE-801; SensoNor, Horten, Norway) with a resonance frequency of $10 \mathrm{kHz}$, whereas the other end was attached to a servomotor (model 308B; Aurora Scientific, Aurora, ON, Canada) with a step time of $250 \mu \mathrm{s}$. In relaxing solution, sarcomere length was set at $2.4 \mu \mathrm{m}$ as the optimal length for force generation $(14,15)$ with the use of a calibrated eyepiece micrometer. Muscle fiber length $(\sim 1-$ $1.5 \mathrm{~mm}$ ) was measured using a reticule in the microscope eyepiece ( $\times 10$ Olympus Plan 10,0.30 numerical aperture [NA]). The $X Y$ fiber diameter (width) was measured with a $\times 40$ objective $(\times 40$ Olympus Plan 40, 0.60 NA). The $\times 40$ objective also was used to measure the $X Z$ fiber diameter (depth) by noting the displacement of the microscope's objective while focusing on the top and bottom surfaces of the fiber. Three width and depth measurements were made along the length of the fiber, and the average values were used to calculate the fiber crosssectional area, assuming that the fiber was ellipsoid in shape.

\section{Composition of Solutions for Single Fiber Measurements}

Concentrated stock solutions of levosimendan (a kind gift from Orion Pharma, Espoo, Finland) were prepared in dimethyl solfoxide (DMSO) and stored at $-20^{\circ} \mathrm{C}$. Before use, levosimendan stock solutions were diluted with experimental solutions. The final concentration of DMSO did not exceed $0.02 \%$. Control experimental solutions contained $0.02 \%$ DMSO (vehicle). Relaxing solution consisted of $1.0 \mathrm{mM} \mathrm{MgCl}_{2}, 4.0 \mathrm{mM}$ $\mathrm{Na}_{2}$ ATP, $5 \mathrm{mM}$ ethyleneglycol-bis-( $\beta$-aminoethyl ether)- $N, N^{\prime}$-tetraacetic acid (EGTA), $10 \mathrm{mM}$ imidazole, $15 \mathrm{mM}$ creatine phosphate, and

TABLE 1. PATIENT CHARACTERISTICS

\begin{tabular}{|c|c|c|c|c|c|c|c|}
\hline Patients & $\begin{array}{l}\text { Age } \\
(y r)\end{array}$ & $\begin{array}{c}\text { BMI } \\
\left(\mathrm{kg} / \mathrm{m}^{2}\right)\end{array}$ & $\begin{array}{c}\mathrm{FEV}_{1}(\% \\
\text { predicted) }\end{array}$ & $\begin{array}{c}\text { VC ( } \% \\
\text { predicted) }\end{array}$ & $\begin{array}{c}\mathrm{FEV}_{1} / \mathrm{VC} \\
(\%)\end{array}$ & $\begin{array}{c}\text { TLC (\% } \\
\text { predicted) }\end{array}$ & $\begin{array}{c}\text { D.co/VA (\% } \\
\text { predicted) }\end{array}$ \\
\hline \multicolumn{8}{|c|}{ Non-COPD } \\
\hline Female & 59 & 23 & 83 & 102 & 74 & 88 & 87 \\
\hline Male & 61 & 27 & 106 & 110 & 73 & 118 & 98 \\
\hline Female & 50 & 21 & 98 & 120 & 72 & 115 & 45 \\
\hline Female & 63 & 23 & 126 & 139 & 74 & 108 & 87 \\
\hline Male & 66 & 21 & 83 & 79 & 78 & 77 & 113 \\
\hline \multicolumn{8}{|l|}{ COPD } \\
\hline Male & 73 & 24 & 86 & 125 & 68 & 112 & 102 \\
\hline Male & 62 & 24 & 60 & 114 & 52 & 111 & 73 \\
\hline Female & 61 & 21 & 54 & 105 & 42 & 124 & 64 \\
\hline Male & 60 & 27 & 85 & 104 & 61 & 106 & 91 \\
\hline Male & 44 & 23 & 29 & 90 & 26 & 133 & 51 \\
\hline
\end{tabular}

Definition of abbreviations: $\mathrm{BMI}=$ body mass index; $\mathrm{D} \mathrm{L}_{\mathrm{LO}} / \mathrm{VA}=$ carbon monoxide transfer coefficient per alveolar volume. sufficient KCL to adjust the total ionic strength to $150 \mathrm{mM}$ at $\mathrm{pH}$ 7.0. The negative logarithm of the free $\mathrm{Ca}^{2+}$ concentration (pCa) of the relaxing solution was 9.0, whereas in activating solutions the $\mathrm{pCa}$ ranged from 7.0 to 4.5 (with maximal activation at pCa 4.5). Sufficient $\mathrm{CaCl}_{2}$ was added to achieve appropriate $\mathrm{pCa}$ in activating solutions.

\section{Diaphragm Muscle Single Fiber Experimental Protocol}

The response of five COPD diaphragm fibers to different doses of levosimendan was determined during submaximal activation (at $\mathrm{pCa}$ 5.8). Figure 1 shows that levosimendan enhanced submaximal force generation with a maximal effect at $10 \mu \mathrm{M}$, which is in accordance with previously reported dose-response curves for cardiac fibers (10). Accordingly, further experiments were conducted with either levosimendan $(10 \mu \mathrm{M})$ or vehicle. Maximal force was determined by perfusing the experimental chamber with solutions of, successively, pCa 9 and pCa 4.5. Then pCa 9.0 solution was perfused through the chamber to relax the fiber. Subsequently, fibers were perfused with solutions containing incremental $\mathrm{Ca}^{2+}$ concentrations. Each time a plateau was reached, the next $\mathrm{pCa}$ solution was perfused through the experimental chamber. To improve functional stability of muscle fibers, we applied Brenner cycling (16), as modified by Sweeney and colleagues (17), throughout the experimental protocol. Accordingly, no functional decline in maximal force generation over time was observed. MIDAC software (Radboud University, Nijmegen, The Netherlands) and a data-acquisition board were used to record signals.

\section{Diaphragm Muscle Single Fiber Contractile Determinations}

Maximum specific force was determined by dividing the isometric force generated at pCa 4.5 by cross-sectional area (CSA). The force-pCa relationship was derived by plotting recorded force against $\mathrm{pCa}$ of the corresponding activating solution. Graphpad software (Graphpad Software Inc., San Diego, CA) was used to calculate the $\mathrm{Ca}^{2+}$ concentration required for half-maximum activation, $\mathrm{pCa}_{50}(\mathrm{pCa}=$ $\left.{ }^{-10} \log \left[\mathrm{Ca}^{2+}\right]\right)$, as an index of $\mathrm{Ca}^{2+}$ sensitivity of force generation and the Hill coefficient, as a measure of myofilament cooperativity (18).

\section{Myosin Heavy Chain Isoform Composition Determination}

Because $\mathrm{pCa}_{50}$ is significantly different between slow and fast diaphragm fibers (19), diaphragm fibers were designated as slow or fast on the basis of myosin heavy chain isoform determination. Determination of myosin heavy chain isoform composition by SDS-PAGE was described previously (13).

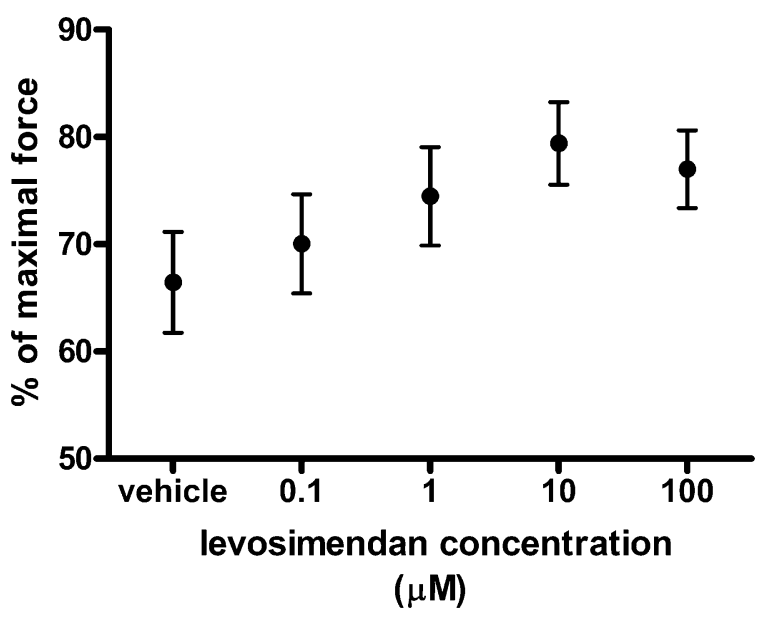

Figure 1. Dose-response effects of levosimendan on diaphragm fiber submaximal force-generating capacity. Incremental levosimendan concentrations were added to diaphragm fibers $(n=5)$ from patients with chronic obstructive pulmonary disease during sub-maximal activation at $\mathrm{pCa}$ 5.8. Force generation increases with rising levosimendan concentration, with a maximal effect at $10 \mu \mathrm{M}$. 


\section{Data Treatment and Statistical Methods}

Differences regarding force-pCa relations between groups were statistically analyzed with a two-way repeated measures analysis of variance (ANOVA) test with $\mathrm{pCa}$ as the repeated measures factor and the presence of levosimendan as the second factor. One-way ANOVA with post hoc Student-Newman-Keuls testing was performed to evaluate the statistical significance of differences between groups of other contractile characteristics. A probability level of $P<0.05$ was considered significant. Mean \pm SEM values are presented in text, tables, and figures.

Sample size of 25 fibers (i.e., 12-13 per treatment group), calculated a priori, was based upon the assumption to detect an increase of submaximal force of at least $15 \%$ by $10 \mu \mathrm{M}$ levosimendan (10) with a standard deviation of $15 \%$ (5), $80 \%$ probability, and an $\alpha$ level of 0.05 . Because we determined the effect in both slow and fast fibers, a total of 100 diaphragm fibers obtained from five patients with COPD and five non-COPD patients were measured (i.e., 10 fibers per patient; five with $10 \mu \mathrm{M}$ levosimendan and five with vehicle).

\section{RESULTS}

\section{Patient Characteristics}

Patient characteristics and pulmonary function data are shown in Table 1. Based on Global Initiative for Chronic Obstructive Lung Disease (GOLD) classification, two patients had mild COPD (stage I), two patients had moderate COPD (stage II) and one patient was classified as very severe COPD (stage IV). One patient without COPD had a mild restriction, and another patient without COPD had a reduced lung diffusion capacity.

\section{Diaphragm Fiber Contractility}

Levosimendan increased calcium sensitivity of force generation in diaphragm fibers from patients with and without COPD, which is indicated by a leftward shift of the force-pCa relation in levosimendan-exposed fibers (Figure 2). During submaximal activation, for instance at pCa 6.0, levosimendan increased diaphragm fiber force by 20 to $30 \%$ compared with vehicleexposed fibers. Accordingly, the $\mathrm{pCa}_{50}$, a measure for calcium sensitivity of force generation, is higher in levosimendan-treated fibers than in vehicle-treated fibers for both COPD and nonCOPD diaphragm fibers (Figure 3). Separation of the results into fibers expressing slow or fast myosin heavy chain showed that levosimendan's effect was more pronounced in slow fibers (Figure 3A) than in fast fibers (Figure 3B). Myofilament cooperativity, indicated by the Hill coefficient, did not differ between vehicle and levosimendan-treated fibers; for slow nonCOPD fibers $(1.47 \pm 0.11$ vs. $1.77 \pm 0.22)$, for slow COPD fibers $(1.55 \pm 0.16$ vs. $1.30 \pm 0.13)$, for fast non-COPD fibers $(2.85 \pm$ 0.18 vs. $3.09 \pm 0.73)$ and for fast COPD fibers $(3.18 \pm 0.56$ vs. $2.02 \pm 0.18)$. Also, levosimendan did not significantly affect diaphragm fiber maximal force generation in both COPD and non-COPD fibers (Figure 4).

\section{COPD Versus Non-COPD}

The effect size of levosimendan on calcium sensitivity did not differ between COPD and non-COPD diaphragm fibers, for both slow and fast typed fibers (Figure 3). Vehicle-treated COPD fibers displayed lower calcium sensitivity and maximal force-generating capacity compared with vehicle-treated fibers from patients without COPD (Figures 3 and 4), which confirms our previously published data (5).

\section{DISCUSSION}

The present study is the first to show that pharmacological modulation of the troponin complex improves human diaphragm muscle fiber contractility. Recently, we have shown that calcium sensitivity of force generation in the diaphragm of patients with COPD is impaired (5). Data of the present study confirm these findings and show that levosimendan improves calcium sensitivity in diaphragm muscle fibers of these patients. Interestingly, levosimendan also improved calcium sensitivity of diaphragm fibers from the diaphragm of patients without COPD. Under certain conditions, calcium sensitization offers a promising mechanism for the treatment of diaphragm weakness, especially in patients with COPD.

Respiratory failure is a prominent manifestation in COPD and is characterized by an increased energy demand on weakened respiratory muscles (1). Increasing the workload on weakened respiratory muscle leads to reduced efficiency of respiratory muscles, which further restricts the limited energy reserves of the diseased body $(20,21)$. To break this viscous circle it is therefore clinically relevant to seek therapies that improve the efficiency of respiratory muscles. Levosimendan has been developed in attempts to find solutions for similar questions regarding cardiac muscle (22). The positive inotropic effects of levosimendan are thought to result mainly from calcium sensitization of cardiac muscle fibers (23). In vivo, both the heart and diaphragm muscle undergo repetitive cycles of contraction and relaxation. This requires continuous sequestration of calcium at the muscle fiber level, which is an energy-consuming process that comprises 30 to $40 \%$ of total muscle energy consumption during normal contractions (24). Current and previous (5) data show that more calcium is needed in diaphragm fibers from patients with COPD to generate the same amount of force as patients without COPD. Accordingly, the stimulation frequency of diaphragm fibers should be increased. Indeed, discharge frequency of motor neurons that activate the diaphragm is twofold higher in patients with COPD during resting breathing (25). This will however impose a higher burden on the calcium sequestration, rendering diaphragm performance less efficient. Conversely, improvement of diaphragm efficiency could be established by generating the same amount of force with less calcium (i.e., increasing the sensitivity of myofilaments for calcium). The present study demonstrates that levosimendan does increase the calcium sensitivity of diaphragm fibers from patients with COPD.

\section{Mechanisms of Action}

In cardiac muscle, levosimendan is presumed to increase calcium sensitivity by stabilizing the conformation of the troponin complex, which triggers muscle contraction (26). Indeed, troponin C has been shown to interact with levosimendan in cardiac muscle (11). Two isoforms of troponin $\mathrm{C}$ exist in muscle tissue: the cardiac/slow isoform present in cardiac and slow-type skeletal muscle, and the fast isoform present in fast-type skeletal muscles. The present finding, that levosimendan improves contractility of slow diaphragm fibers, can be explained by its interaction with cardiac/slow troponin C. In addition, we observed that levosimendan enhances fast diaphragm fiber contractility. Fast skeletal and slow skeletal troponin $\mathrm{C}$ are approximately $70 \%$ identical (27), but whether levosimendan can also bind to fast troponin C has, as far as we know, never been described. Our observations in fast diaphragm fibers suggest a possible interaction of levosimendan with fast troponin C. Another possibility is that some fibers express fast myosin heavy chain with slow troponin C. Increased expression of slow troponin $\mathrm{C}$ has previously been demonstrated in the diaphragm of patients with COPD (12). It was postulated that this results from a reduction of fast fibers rather than an altered isoform expression within fast fibers. Our results support this postulation, because the effect size of levosimendan on COPD fibers was similar to its effect size on non-COPD fibers. Furthermore, adult skeletal muscle generally coexpresses fast 
A

Myosin Heavy Chain Slow

Non-COPD

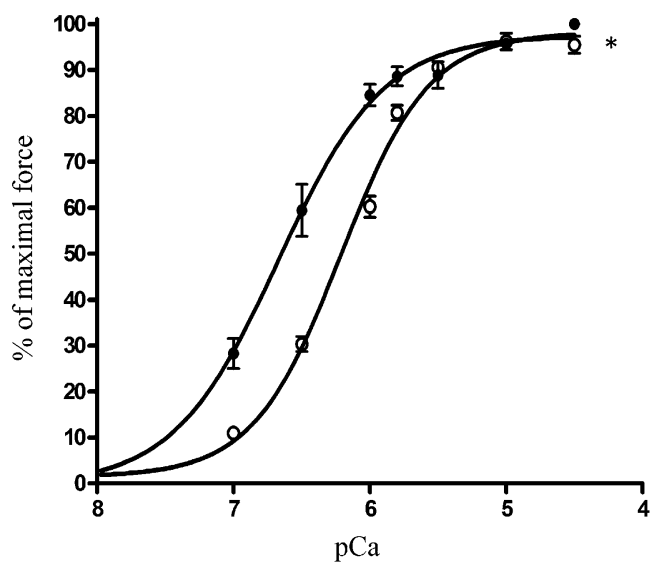

- Vehicle $(n=16)$

- $10 \mu \mathrm{M}$ levosimendan $(\mathrm{n}=16)$

B

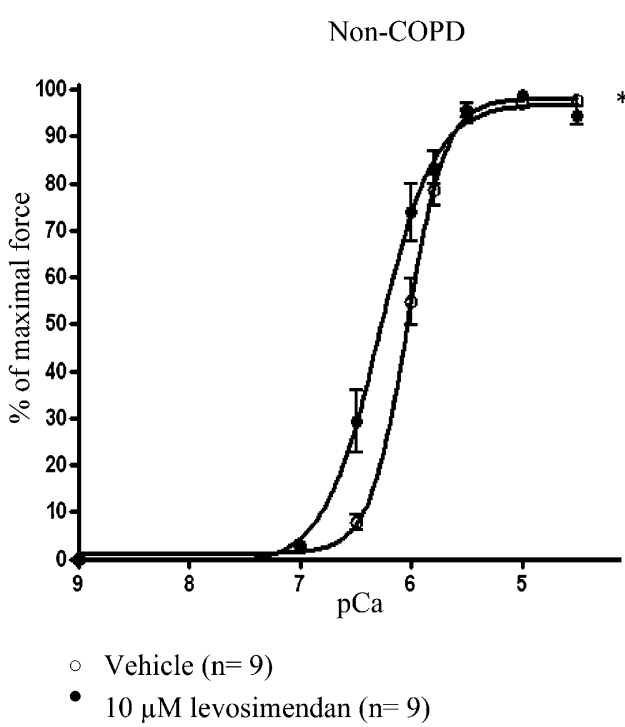

Myosin Heavy Chain Fast

- Vehicle $(n=17)$

- $10 \mu \mathrm{M}$ levosimendan $(\mathrm{n}=13)$

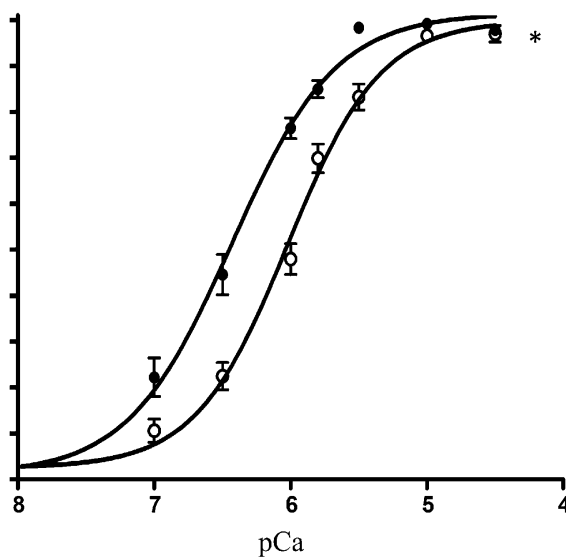

Figure 2. Force-calcium characteristics of diaphragm fibers from patients with and without chronic obstructive pulmonary disease in the presence of $10 \mu \mathrm{M}$ levosimendan or vehicle activating solutions. Isometric force generation in response to solutions with incremental calcium concentrations was determined. Submaximal force generation is higher in diaphragm fibers treated with levosimendan when compared with vehicle. *Twoway repeated measures analysis showed that force-pCa relations of fibers treated with levosimendan were significantly different from force-pCa relations of fibers treated with vehicle, for both slow and fast fibers ( $A$ and $B$, respectively). Data are presented as means \pm SEM. myosin heavy chain with fast troponin C (28), so we do not expect that expression of slow troponin $\mathrm{C}$ explains the effect of levosimendan in fast fibers. Nevertheless, the effect of levosimendan on $\mathrm{pCa}_{50}$ was more profound in slow fibers compared with fast fibers (Figure 2), indicating that the effect size of levosimendan depends on the isoform expression of myofibrillar proteins. A recent study showed that specific structures in troponin I influence the binding of levosimendan to the troponin complex. It could therefore be that the effect size of levosimendan is modulated by isoforms of different myofibrillar proteins. As expression of type I fibers in the diaphragm of patients with COPD is increased (12) levosimendan treatment may be more effective in patients with COPD than in healthy subjects. Levosimendan did not affect maximal force generation, which implies that the maximal amount of attached cross-bridges as well as force per cross-bridge was not affected by levosimendan.

\section{Study Limitations}

The effect of levosimendan on diaphragm muscle fibers was tested in vitro, which brings several advantages. Using skinned muscle fibers we were able to specifically study the effects of levosimendan on contractile protein function. This would not be possible in vivo because measured force in vivo depends on many other factors including patient motivation, neuromuscular transmission, and intracellular calcium signaling. In addition, the cardiovascular effects of levosimendan may affect respiratory muscle function in patients with COPD. Thus, skinned diaphragm fibers provide an excellent model to study the effects of levosimendan on diaphragm contractility. The data of the present study provide a strong rationale to test the effects of calcium sensitizers on diaphragm muscle function in vivo under appropriate conditions.

The levosimendan concentration used in the present study $(10 \mu \mathrm{M})$ was derived from dose-response study and was in line with previous data from cardiac muscle fibers (10). Although $0.1 \mu \mathrm{M}$ levosimendan resulted in enhanced force generation, the dose-response curve on COPD diaphragm fibers revealed that $10 \mu \mathrm{M}$ levosimendan induces a maximal effect on force improvement. This concentration is slightly higher than plasma concentrations measured after infusion using clinically recom- 
A

Myosin Heavy Chain Slow

$\square$ Vehicle

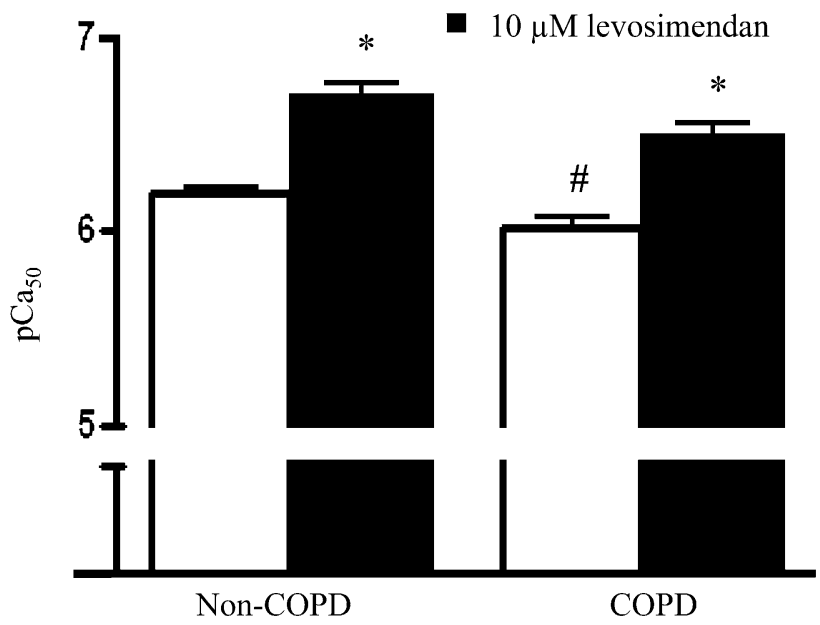

B

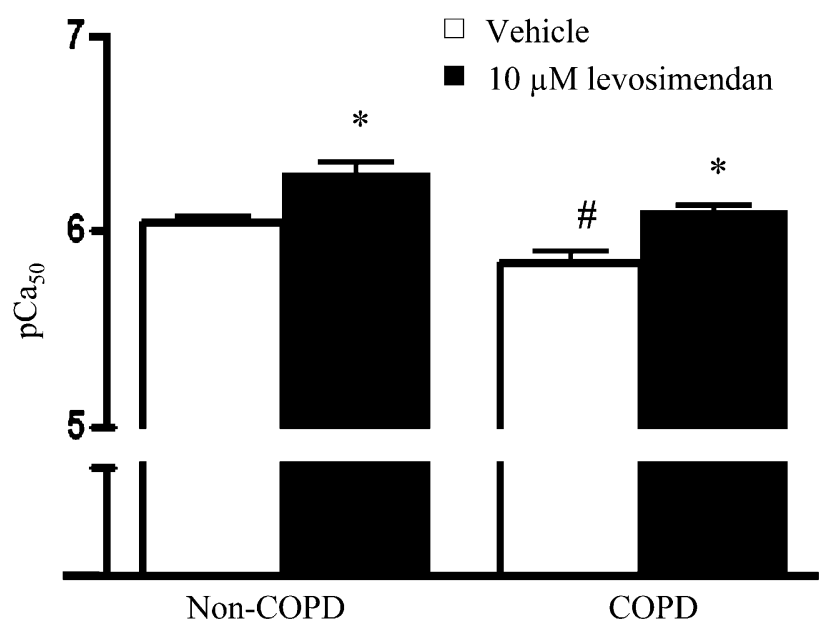

Figure 3. Values of $\mathrm{pCa}_{50}$, an index for calcium sensitivity of force generation, from diaphragm fibers of patients with and without chronic obstructive pulmonary disease (COPD) in the presence of $10 \mu \mathrm{M}$ levosimendan or vehicle solution. Calcium sensitivity is lower in COPD fibers than in non-COPD fibers. Diaphragm fibers treated with levosimendan display higher calcium sensitivity than fibers treated with vehicle, for both slow and fast fibers ( $A$ and $B$, respectively). Data are presented as means \pm SEM. ${ }^{*} P<0.01$ compared with vehicle-treated fibers within subject group; ${ }^{P} P<0.05$ compared with vehicle treated non-COPD fibers within fiber-type group. $\mathrm{pCa}_{50}=-{ }^{10} \log \left[\mathrm{Ca}^{2+}\right]$ at which $50 \%$ of maximal force is generated.

mended dosages (24-h infusion with $0.1-0.4 \mu \mathrm{g} / \mathrm{kg} / \mathrm{min}$ ) (29). Furthermore, intracellular levosimendan concentrations seem to be more relevant and could exceed blood values. Local concentrations are, however, difficult to determine because in vivo levosimendan has a short half-life $(\sim 1 \mathrm{~h})$ as it is reduced to OR-1855 by intestinal bacteria (30). OR-1855 is further metabolized by acetylation to OR-1896, which has similar calcium sensitizing properties as levosimendan (23), but a much longer half-life (30).

\section{Clinical Implications}

The pathophysiology of weaning failure from mechanical ventilation is complex and multifactorial (31). However, it has been
A

Myosin Heavy Chain Slow

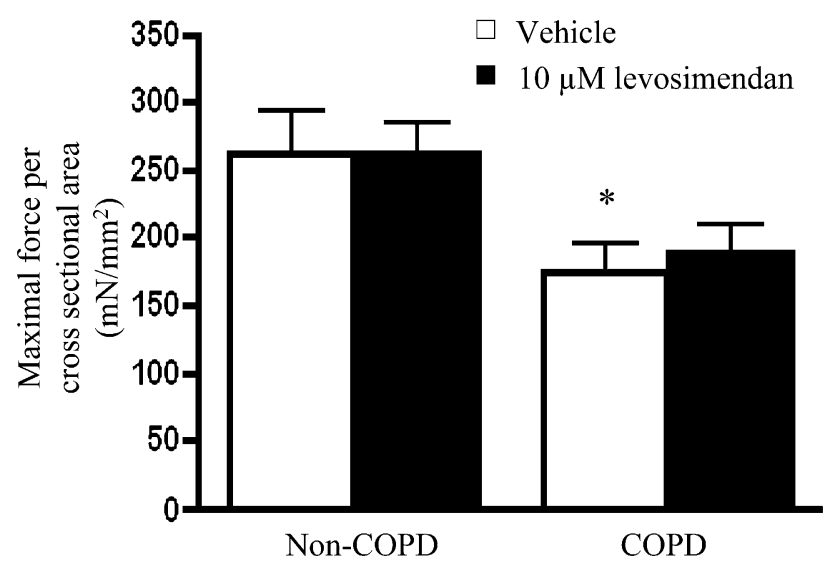

B

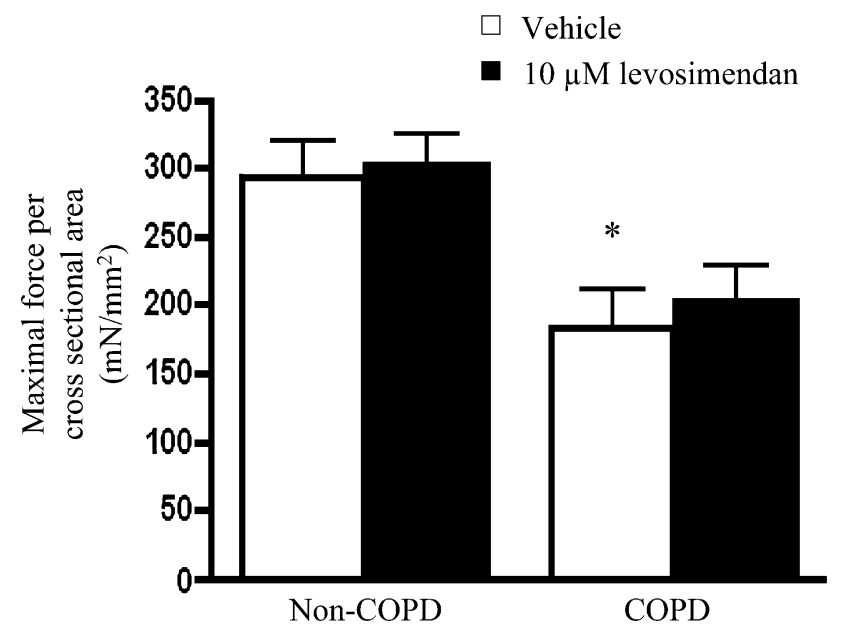

Figure 4. Maximal force generation of diaphragm fibers from patients with and without chronic obstructive pulmonary disease (COPD) in the presence of $10 \mu \mathrm{M}$ levosimendan or vehicle solution. COPD diaphragm fibers generated lower maximal forces than non-COPD fibers, in both slow and fast fibers (Figures $4 A$ and $4 B$, respectively). Maximal force generation is not significantly different between levosimendan and vehicle-treated fibers within patient groups. Data are presented as means \pm SEM. ${ }^{*} P<0.05$ compared with vehicle-treated non-COPD fibers within fiber-type group.

recognized that, in patients with COPD, maximal inspiratory pressure is significantly lower at the time of weaning failure than at the time of successful weaning (32). In addition, patients with severe COPD failing to wean from mechanical ventilation generate lower inspiratory transdiaphragmatic pressure than patients without severe COPD (33). Accordingly, improving respiratory muscle contractility may contribute to successful weaning from mechanical ventilation in these patients. Moreover, as levosimendan also improves contractility in diaphragm fibers from patients without COPD, this agent may appear beneficial to other patients difficult to wean from mechanical ventilation. The results of the present study provide an excellent rationale to test the efficacy of levosimendan in this clinical setting. However, it should be kept in mind that in vivo levosimendan affects other cell processes besides calcium sensitivity. For instance, opening of ATP-sensitive potassium channels in vascular smooth muscle affects pulmonary and systemic vascular 
resistance, resulting in reduction in mean arterial blood pressure (34). The final effects of levosimendan on cardiac contractility, respiratory muscle function, and oxygen transport in non-heartfailure patients are difficult to predict but should be answered by clinical studies. By increasing $\mathrm{pCa}_{50}$ levosimendan could improve respiratory muscle function during weaning without increasing energy expenditure. The latter is important because respiratory muscles could fatigue during weaning trails $(32,35)$. A major cause of fatigue is the accumulation of inorganic phosphate resulting from the excessive breakdown of phosphocreatine during periods of high energy demand (36). Elevated inorganic phosphate levels depress calcium sensitivity (37), which evidently impairs contractile muscle function. Hence, levosimendan seems highly appropriate to counteract fatigue-induced muscle weakness because it enhances the sensitivity for calcium without increasing energy demand. Very recently, Levine and colleagues showed that controlled mechanical ventilation induces rapid atrophy of human diaphragm muscle fibers (38). Although not studied, it is likely that atrophy will result in impaired diaphragm contractility in these patients. Therefore, levosimendan may appear beneficial in difficult mechanical ventilation weaning of patients without COPD as well. Our study demonstrated that levosimendan increased calcium sensitivity of diaphragm fibers from subjects without COPD.

Levosimendan is generally well tolerated by patients and hypotension is one of the most observed side effects but is not common when the treatment remains within the recommended dose. If necessary, hypotension can be counteracted by low-dose norepinephrine $(39,40)$.

In conclusion, the present study demonstrates that levosimendan enhances the force-generating capacity of diaphragm fibers from patients with and without COPD by increasing calcium sensitivity of force generation. The results of our study provide a strong rationale for testing the effect of calcium sensitizers on respiratory muscle contractility in patients with COPD during elevated loading, such as weaning from mechanical ventilation.

Conflict of Interest Statement: None of the authors has a financial relationship with a commercial entity that has an interest in the subject of this manuscript.

Acknowledgments: The authors thank Dr. J. Levijoki from Orion Pharma, Espoo, Finland, for generously providing levosimendan; Prof. Dr. H.A. van Swieten and Dr. A.F.T.M. Verhagen from the Department of Cardiothoracic Surgery, Radboud University Nijmegen Medical Centre, for collecting the diaphragm muscle biopsies; and M. Linkels from the Department of Pulmonary Diseases, Radboud University Nijmegen Medical Centre, for the determination of myosin heavy chain isoforms.

\section{References}

1. Laghi F, Tobin MJ. Disorders of the respiratory muscles. Am J Respir Crit Care Med 2003;168:10-48.

2. Ottenheijm CA, Heunks LM, Dekhuijzen PN. Diaphragm muscle fiber dysfunction in chronic obstructive pulmonary disease: toward a pathophysiological concept. Am J Respir Crit Care Med 2007;175:1233-1240.

3. Gray-Donald K, Gibbons L, Shapiro SH, Macklem PT, Martin JG. Nutritional status and mortality in chronic obstructive pulmonary disease. Am J Respir Crit Care Med 1996;153:961-966.

4. Nava S, Rubini F, Zanotti E, Ambrosino N, Bruschi C, Vitacca M, Fracchia C, Rampulla C. Survival and prediction of successful ventilator weaning in COPD patients requiring mechanical ventilation for more than 21 days. Eur Respir J 1994;7:1645-1652.

5. Ottenheijm CA, Heunks LM, Sieck GC, Zhan WZ, Jansen SM, Degens H. de BT, Dekhuijzen PN. Diaphragm dysfunction in chronic obstructive pulmonary disease. Am J Respir Crit Care Med 2005; 172:200-205.

6. Ottenheijm CA, Heunks LM, Hafmans T, van der Ven PF, Benoist C, Zhou H, Labeit S, Granzier HL, Dekhuijzen PN. Titin and diaphragm dysfunction in chronic obstructive pulmonary disease. Am J Respir Crit Care Med 2006;173:527-534.
7. Ottenheijm CA, Heunks LM, Li YP, Jin B, Minnaard R, van Hees HW, Dekhuijzen PN. Activation of the ubiquitin-proteasome pathway in the diaphragm in chronic obstructive pulmonary disease. Am J Respir Crit Care Med 2006;174:997-1002.

8. Haikala H, Nissinen E, Etemadzadeh E, Levijoki J, Linden IB. Troponin C-mediated calcium sensitization induced by levosimendan does not impair relaxation. J Cardiovasc Pharmacol 1995;25:794-801.

9. Follath F, Cleland JG, Just H, Papp JG, Scholz H, Peuhkurinen K, Harjola VP, Mitrovic V, Abdalla M, Sandell EP, et al. Efficacy and safety of intravenous levosimendan compared with dobutamine in severe low-output heart failure (the LIDO study): a randomised double-blind trial. Lancet 2002;360:196-202.

10. Edes I, Kiss E, Kitada Y, Powers FM, Papp JG, Kranias EG, Solaro RJ. Effects of levosimendan, a cardiotonic agent targeted to troponin C, on cardiac function and on phosphorylation and $\mathrm{Ca}^{2+}$ sensitivity of cardiac myofibrils and sarcoplasmic reticulum in guinea pig heart. Circ Res 1995;77:107-113.

11. Sorsa T, Pollesello P, Permi P, Drakenberg T, Kilpelainen I. Interaction of levosimendan with cardiac troponin $\mathrm{C}$ in the presence of cardiac troponin I peptides. J Mol Cell Cardiol 2003;35:1055-1061.

12. Levine S, Kaiser L, Leferovich J, Tikunov B. Cellular adaptations in the diaphragm in chronic obstructive pulmonary disease. $N$ Engl J Med 1997:337:1799-1806.

13. van Hees HW, van der Heijden HF, Ottenheijm CA, Heunks LM, Pigmans CJ, Verheugt FW, Brouwer RM, Dekhuijzen PN. Diaphragm single-fiber weakness and loss of myosin in congestive heart failure rats. Am J Physiol Heart Circ Physiol 2007;293:819-828.

14. Zuurbier CJ, Heslinga JW, Lee-de Groot MB, van der Laarse WJ. Mean sarcomere length-force relationship of rat muscle fibre bundles. J Biomech 1995;28:83-87.

15. Burkholder TJ, Lieber RL. Sarcomere length operating range of vertebrate muscles during movement. J Exp Biol 2001;204:1529-1536.

16. Brenner B. Technique for stabilizing the striation pattern in maximally calcium-activated skinned rabbit psoas fibers. Biophys $J$ 1983;41:99102.

17. Sweeney HL, Corteselli SA, Kushmerick MJ. Measurements on permeabilized skeletal muscle fibers during continuous activation. Am J Physiol 1987;252:C575-C580.

18. Donaldson SK, Kerrick WG. Characterization of the effects of $\mathrm{Mg}^{2+}$ on $\mathrm{Ca}^{2+}$ - and $\mathrm{Sr}^{2+}$-activated tension generation of skinned skeletal muscle fibers. J Gen Physiol 1975;66:427-444.

19. Geiger PC, Cody MJ, Sieck GC. Force-calcium relationship depends on myosin heavy chain and troponin isoforms in rat diaphragm muscle fibers. J Appl Physiol 1999;87:1894-1900.

20. Jubran A, Tobin MJ. Pathophysiologic basis of acute respiratory distress in patients who fail a trial of weaning from mechanical ventilation. Am J Respir Crit Care Med 1997;155:906-915.

21. Laghi F, Jubran A, Topeli A, Fahey PJ, Garrity ER Jr, Arcidi JM, de Pinto DJ, Edwards LC, Tobin MJ. Effect of lung volume reduction surgery on neuromechanical coupling of the diaphragm. Am J Respir Crit Care Med 1998;157:475-483.

22. Kass DA, Solaro RJ. Mechanisms and use of calcium-sensitizing agents in the failing heart. Circulation 2006;113:305-315.

23. Szilagyi S, Pollesello P, Levijoki J, Kaheinen P, Haikala H, Edes I, Papp Z. The effects of levosimendan and OR-1896 on isolated hearts, myocyte-sized preparations and phosphodiesterase enzymes of the guinea pig. Eur J Pharmacol 2004;486:67-74.

24. Barclay CJ, Woledge RC, Curtin NA. Energy turnover for $\mathrm{Ca}^{2+}$ cycling in skeletal muscle. J Muscle Res Cell Motil 2007;28:259-274.

25. De TA, Leeper JB, McKenzie DK, Gandevia SC. Neural drive to the diaphragm in patients with severe COPD. Am J Respir Crit Care Med 1997;155:1335-1340.

26. Sorsa T, Pollesello P, Solaro RJ. The contractile apparatus as a target for drugs against heart failure: interaction of levosimendan, a calcium sensitiser, with cardiac troponin-C. Mol Cell Biochem 2004;266:87-107.

27. Roher A, Lieska N, Spitz W. The amino acid sequence of human cardiac troponin-C. Muscle Nerve 1986;9:73-77.

28. Dhoot GK, Perry SV. Distribution of polymorphic forms of troponin components and tropomyosin in skeletal muscle. Nature 1979;278: 714-718.

29. Kivikko M, Lehtonen L, Colucci WS. Sustained hemodynamic effects of intravenous levosimendan. Circulation 2003;107:81-86.

30. Antila S, Sundberg S, Lehtonen LA. Clinical pharmacology of levosimendan. Clin Pharmacokinet 2007;46:535-552.

31. Vassilakopoulos T, Zakynthinos S, Roussos C. Respiratory muscles and weaning failure. Eur Respir J 1996;9:2383-2400. 
32. Vassilakopoulos T, Zakynthinos S, Roussos C. The tension-time index and the frequency/tidal volume ratio are the major pathophysiologic determinants of weaning failure and success. Am J Respir Crit Care Med 1998;158:378-385.

33. Jubran A. Critical illness and mechanical ventilation: effects on the diaphragm. Respir Care 2006;51:1054-1061.

34. Michaels AD, McKeown B, Kostal M, Vakharia KT, Jordan MV, Gerber IL, Foster E, Chatterjee K. Effects of intravenous levosimendan on human coronary vasomotor regulation, left ventricular wall stress, and myocardial oxygen uptake. Circulation 2005;111:15041509.

35. Littleton SW, Laghi F, Sinderby C, Beck J, Tobin MJ, Jubran A. Can patients who fail a weaning trial develop short-lasting diaphragmatic fatigue? [abstract]. Am J Respir Crit Care Med 2008;177: A375.

36. Allen DG, Lamb GD, Westerblad H. Skeletal muscle fatigue: cellular mechanisms. Physiol Rev 2008;88:287-332.
37. Millar NC, Homsher E. The effect of phosphate and calcium on force generation in glycerinated rabbit skeletal muscle fibers: a steady-state and transient kinetic study. J Biol Chem 1990;265:20234-20240.

38. Levine S, Nguyen T, Taylor N, Friscia ME, Budak MT, Rothenberg P, Zhu J, Sachdeva R, Sonnad S, Kaiser LR, et al. Rapid disuse atrophy of diaphragm fibers in mechanically ventilated humans. $N$ Engl J Med 2008;358:1327-1335.

39. Nieminen MS, Akkila J, Hasenfuss G, Kleber FX, Lehtonen LA, Mitrovic V, Nyquist O, Remme WJ. Hemodynamic and neurohumoral effects of continuous infusion of levosimendan in patients with congestive heart failure. J Am Coll Cardiol 2000;36:1903-1912.

40. Moiseyev VS, Poder P, Andrejevs N, Ruda MY, Golikov AP, Lazebnik LB, Kobalava ZD, Lehtonen LA, Laine T, Nieminen MS, et al. Safety and efficacy of a novel calcium sensitizer, levosimendan, in patients with left ventricular failure due to an acute myocardial infarction: a randomized, placebo-controlled, double-blind study (RUSSIAN). Eur Heart J 2002;23:1422-1432. 